\title{
Estrategia didáctica y el empleo de TIC para el desarrollo de la comprensión lectora en los estudiantes de 6to año de la Educación Básica General en Ecuador
}

Didactic strategy and the use of ICT for the development of reading comprehension in 6th year students of General Basic Education in Ecuador

Estrategia didáctica y el empleo de TIC

\section{Tania Elizabeth Chávez Lucas Lcda. ${ }^{(1)}$}

Ing. Jimmy Manuel Zambrano Acosta PhD ${ }^{(2)}$

Luis Santiago Quiroz Fernández PhD ${ }^{(3)}$

${ }^{1}$ Estudiante del Programa de Maestría Académica con trayectoria en investigación en Comunicación Educativa del Instituto de Posgrado de la Universidad Técnica de Manabí, Ecuador

${ }^{2}$ Doctor en Ciencias de la Educación Profesor del Instituto de Posgrado de la Universidad Técnica de Manabí, Ecuador.

${ }^{3}$ Doctor en Ciencias Técnicas de la Universidad Tecnológica de la Habana "José Antonio Echeverria" Cuba, Director de Posgrado de la Universidad Técnica de Manabí, Ecuador.

\section{Resumen}

El presente versa sobre un estudio realizado en función del desarrollo del aprendizaje de los estudiantes de 6to año de la Educación General Básica en el desarrollo de la comprensión lectora y el empleo de TIC en el proceso. El tema resulta de mucho interés dado que en los informes realizados por las autoridades regionales y del territorio, uno de los principales problemas en el área del español y la literatura es precisamente la comprensión lectora, lo cual se efectúa en todas las asignaturas, denotando su carácter transdisciplinar. Sobre la base de las insuficiencias detectadas en una muestra de 25 estudiantes, se determinó como problema científico a tratar: Limitaciones en la comprensión lectora de los estudiantes de sexto año de Educación General Básica de la Escuela "Medardo Alfaro de Montecristi”, Manabí, Ecuador. Para ello, se propuso como objetivo general: Elaborar una estrategia didáctica para el desarrollo de la comprensión lectora, mediadas por las TIC para los 
escolares de sexto año de dicho contexto. Desde posturas críticas sistematizadas sobre los procederes metodológicos y didácticos, se determinaron los presupuestos teóricos y metodológicos que, desde una perspectiva de análisis sustentan el desarrollo de la comprensión lectora y el empleo de TIC para favorecer el proceso y garantizar mayor motivación por la lectura. En la investigación se elabora, instrumenta y evalúa una estrategia didáctica para el desarrollo del aprendizaje de la comprensión lectora, que desde los principales y sustanciales resultados logrados se le confiere pertinencia, novedad y actualidad.

Palabras clave: Lectura; Comprensión lectora; Estrategia didáctica; Tecnologías de la Información y las Comunicaciones; Prezi.

\section{Summary}

This is about a study carried out based on the learning development of 6th year students of Basic General Education in the development of reading comprehension and the use of ICT in the process. The subject is of great interest given that in the reports made by regional and territorial authorities, one of the main problems in the area of Spanish and literature is precisely reading comprehension, which is carried out in all subjects, denoting their transdisciplinary character. Based on the shortcomings detected in a sample of 25 students, the following scientific problem to be addressed was determined: Limitations in the reading comprehension of sixth year students of Basic General Education of the "Medardo Alfaro de Montecristi" School, Manabí, Ecuador. For this, it was proposed as a general objective: To develop a didactic strategy for the development of reading comprehension, mediated by ICT for sixth-year schoolchildren in that context. From systematized critical positions on the methodological and didactic procedures, the theoretical and methodological assumptions were determined that, from an analysis perspective, support the development of reading comprehension and the use of ICT to favor the process and guarantee greater motivation for reading. The research elaborates, implements and evaluates a didactic strategy for the development of reading comprehension learning, which, from the main and substantial results achieved, is given relevance, novelty and timeliness.

Keywords: Reading; Reading comprehension; Didactic strategy; Information technology and communications; Prezi. 


\section{Introducción}

La lectura en general y la comprensión lectora en particular, en todos los contextos y desempeños del ser humano en cuanto a ilustrarse y construir conocimientos se refiere, son componentes necesarios e imprescindibles en el desarrollo del que estudia, o simplemente desea conocer, profundizar en aspectos de un determinado campo del saber, ciencia, humanidades o simplemente noticias de sucesos. La lectura para los estudiantes en los diversos niveles educativos, tanto en el ciclo escolar básico como en todas las etapas de su vida académica por las que transitará, ha de ser indispensable y de vital importancia en su crecimiento.

Lamentablemente, no todos los ciudadanos realizan actividades de lectura con asiduidad, en el caso de Ecuador en particular, se evidencia en los resultados obtenidos en la aplicación de diversas evaluaciones realizadas por la UNESCO $(2014,2016,2018)$ en las que ubican al Ecuador rezagado según los índices de lectura de América Latina.

Es conocido que las habilidades logradas en función de la lectura influyen en el proceso de análisis y concentración del sujeto, lo que facilita en mayor medida la autonomía cognitiva, favorece el libre pensamiento y el acervo cultural aumenta en mayor medida. (Tresca, M. 2015). Por lo tanto, a través de la lectura, se construyen nuevos saberes y conocimientos que ayudan al desarrollo intelectual y creativo, por lo que, en el proceso de enseñanza-aprendizaje (PEA) de todos los contenidos necesarios por etapas, la lectura es elemento clave y definitorio para poder alcanzar los mejores índices de rendimiento escolar, sobre la base de la comprensión de los contenidos que aparecen en los documentos que se leen.

De acuerdo a un estudio realizado en Ecuador los resultados de las pruebas nacionales e internacionales indican serias deficiencias en la comprensión de lectura, habilidad escolar fundamental para acceder a las demás disciplinas escolares. La Evaluación Censal de Estudiantes 2007 reporta que sólo el $16 \%$ de estudiantes desde segundo de primaria alcanza el nivel esperado para su grado en comprensión de textos (es decir, llega a ser capaz de deducir el tema central de un texto, establecer relaciones causa-efecto explícitas e identificar datos explícitos) (Ministerio de Educación, 2010). Aunque se observa un progreso en los años siguientes (16, $9 \%$ para el 2008, 23,1\% para el 2009 y 28,7 \% para el 2010) todavía más de la mitad de los escolares de esos años no alcanza estos 
resultados en el aprendizaje de la comprensión lectora (Ministerio de Educación de Ecuador; Ecuador, M. D. 2010).

La realidad descrita anteriormente tiene una estrecha relación con los datos evidenciados en el Ecuador, como son:

a) La inequidad existente en el país, ya que los bajos resultados se acentúan para las escuelas públicas, especialmente en las zonas rurales y más pobres del país (Ministerio de Educación, 2010).

b) Los estudiantes con bajo rendimiento en el inicio de la lectura continúan con dificultades a lo largo de su escolaridad, mientras que aquellos con adecuada preparación y buen inicio en la lectura continúan siendo buenos lectores. (Thorne, et. al (2013).

Por otro lado, en el siglo XXI ha adquirido una especial importancia el tema de la lectura desde TIC. Todas las actividades humanas han estado permeadas por ellas. La lingüística no podía estar ajena, es por ello que se han venido desarrollando, a partir del año 2000, herramientas de comunicación e información como por ejemplo diccionarios en formato electrónico, traductores automáticos, atlas lingüísticos computarizados; entre otros (Echevarría, 2002).

Asimismo, a partir del año 2008, se han presentado diferentes proyectos de investigación que establecen la importancia de TIC en el acto de la comprensión lectora. Las investigaciones por ejemplo: Álvarez, G. (2012), Ariaz, et. Al. (2018), Tamayo, (2018), Celin, \& Mendoza, (2019), Campos, (2020), y otros más, los cuales indican que el uso de TIC favorece estrategias cognitivas como el muestreo, la predicción y la deducción; genera motivación e interés en los escolares por las actividades académicas; optimiza los procesos de lectura; permite que los escolares puedan sacar sus propias conclusiones e inferencias de lo que ha observado; y además, le brinda al estudiante estrategias novedosas en su proceso cognitivo.

En estas investigaciones se conciben las TIC como herramientas didácticas que optimizan los procesos lecto-escriturales en los escolares y posibilitan la interacción entre el docente, el escolar y los ejes conceptuales. 


\section{Desarrollo}

En el sexto año de la Educación General Básica de la Escuela Medardo Alfaro de Montecristi, Manabí, donde se realiza el estudio en cuestión, se manifiesta por parte de los estudiantes insuficiencias y problemas con la actividad particular de la lectura; la cual a juicio de la autora del estudio, a partir de la experiencia que posee en funciones educativas y académicas en esta institución escolar, ha notado que no se realiza la lectura con la sistematización necesaria ni se logra con el nivel reflexivo deseado. Algo que impacta de forma negativa el desarrollo del aprendizaje en muchas de las materias escolares, ya que para comprender los contenidos requieren de ser leídos en los libros y demás materiales complementarios.

El trabajo para la comprensión debe realizarse de manera permanente a través de todas las asignaturas en todos los niveles educativos, donde se hacen explícitos los objetivos que se propone la institución en este sentido.

Se pretende que un egresado de la Educación General Básica sea capaz de leer con corrección, expresividad, fluidez y que demuestre la comprensión adecuada de lo leído. En este sentido, se formulan con precisión los objetivos para los cuales debe trabajarse. Se aspira a que el estudiante sea capaz de:

- Conocer el significado de nuevas palabras.

- Responder preguntas sobre el contenido de la lectura.

- Expresar de qué trata la lectura y contarla brevemente.

- Relacionar el texto con las ilustraciones.

- Realizar actividades de prelectura, lectura y poslectura.

- Buscar información en el texto teniendo en cuenta las dimensiones local, global, intratextual e intertextual.

- Establecer relaciones entre el texto y su contexto.

- Reconocer elementos paratextuales.

- Realizar una lectura literal, inferencial y crítica. 
La estrategia a seguir en el aprendizaje del idioma materno, resulta imprescindible, y sobre todo, en la lectura a los distintos usos que ella tiene en la práctica, enfatizar en sus propósitos sociales, multiculturales, y resolver un problema práctico (hacer una comida, construir un objeto); informarse sobre política, cultura, obtener una información específica (una dirección, el significado de una palabra), o leer por placer o esparcimiento.

Por eso, cada docente ha de desarrollar el campo de sus observaciones y experiencias, procurar que el contenido y los métodos que se apliquen, permitan la asimilación consciente y duradera de los conocimientos, la formación de hábitos y habilidades para lograr la adecuada comprensión de los textos leídos. La observación reiterada y la práctica en el análisis y las síntesis de las lecturas realizadas permiten desarrollar destrezas y habilidades significativas ene se sentido.

A medida que los estudiantes avancen en la lectura y escritura de palabras y oraciones, se acostumbran a distinguir y aplicar las técnicas de la lectura en todos los ejercicios que realizan, los pasos y procedimientos a realizar, y a utilizar determinada TIC en función de la lectura, lo que la hace más amena y los motiva en ese sentido. Específicamente, en aquellos actos de lectura en donde el estudiante se enfrenta solamente a los procesos de codificación y decodificación (aspecto mecánico); este proceder incide en detrimento de una lectura comprensiva.

Esto se traduce en que el docente por su parte, tiene la posibilidad de utilizar más frecuencias de las indicadas o mayor tiempo en y para determinadas clases, cuando el contenido que desarrollan o las necesidades de sus estudiantes lo requieran, en función de profundizar en dicha comprensión.

La estrategia didáctica en cuestión, atiende las etapas conocidas para la compresión lectora y esta se nutre del empleo de TIC de acuerdo a las posibilidades de los docentes para orientar las actividades.

La estrategia se expresa a partir de una serie de formas o vías que posibilitan el tratamiento de los contenidos textuales para la comprensión lectora, y que se expresan y argumentan seguidamente.

\section{A. Textos en desorden (durante).}

Se selecciona un texto, literario o no literario, y se plantean en las presentaciones de PREZI por ejemplo, (puede utilizarse power point, blog, etc...) donde se facilitan los textos a leer solo en partes, 
es decir, en párrafos u oraciones en partes. Los estudiantes forman grupos de acuerdo al número de segmentos y se les pide que en equipo puedan traten de reconstruir el texto, en otra plantilla de la plataforma o herramienta utilizada. La única regla que deben seguir es que deben lograr un orden y la disciplina necesaria para la manipulación de los dispositivos que se utilicen. Esto evitará que los estudiantes comiencen a realizar otras actividades, o que algún estudiante o parte del grupo acaparen el trabajo y excluyan a los demás.

\section{B. Transformación de la narración (después)}

Esta estrategia grupal de forma general estimula y motiva a los estudiantes para que utilicen formas alternativas de crear significados, y es un buen sustituto para las molestas pruebas escritas utilizadas para evaluar la comprensión de una lectura asignada o la simple lectura de un texto para responder determinadas preguntas orales.

Seguidamente de que los estudiantes hayan leído los textos sugeridos y orientados por el profesor, deben construir una narración oral con elementos de apoyo para su presentación pública, lo cual se debe realizar a partir de la utilización del programa Prezi, power point, o a partir de imágenes proyectadas por algún dispositivo tecnológico empleado, en la que a partir de esto, se debe construir una narrativa que se ajustará a una de las que fueron propuestas por el docente para que se identifique por el grupo. Lo esencial es que los estudiantes demuestren que han leído y comprendido el texto orientado, y lo sepan ajustar a las imágenes que el docente también les ofrece de forma aleatoria, sin orden alguno, y así y representen su propia interpretación del mismo y puedan ofrecer una narrativa al respecto.

\section{Las tramas narrativas}

Seguidamente de haber leído un texto, el estudiante tratará de recordar el texto que ha construido y almacenado en la memoria y lo escribirá en un armazón o esquema que puede haber sido confeccionado por el docente o por los propios estudiantes, que igualmente lo pueden hacer de forma interactiva con TIC o en sus propios dispositivos para favorecer la rapidez de la actividad en ese sentido. 
Posteriormente, se procede a estimular a los estudiantes para que comparen si la trama que han escrito coincide con la del relato original, el que permanecerá proyectado para todos los estudiantes en el salón.

\section{Fichas de personajes}

Es una parte de la estrategia muy sencilla, en la que se diseña para centrar la atención de los estudiantes y en la cual las TIC favorecen un juegan un papel significativo en su empleo por parte del docente, lo relacionado con las personalidades de protagonistas específicos de un texto tratado.

Se puede solicitar a los estudiantes que dibujen en una aplicación de su móvil, Tablet. Laptop o una PC en el salón, la silueta de un personaje y después que hagan una lista dentro de la silueta con las

diferentes características de los mismos. Éstas se pueden exponer bajo del título de la historia a la que corresponda. De esta manera se puede visualizar las distintas relaciones que se dan entre los personajes y lo que se ha comprendido de las situaciones que se han dado en el texto y de los procederes de los personajes en específico.

\section{E. Conversación escrita con un personaje}

En este aparte, el estudiante elabora un diálogo escrito con un personaje de un texto leído.

El objetivo de esto es estimular a los estudiantes a que se centren en un personaje específico y traten de comprender su forma de pensar, hablar y escribir, lo mismo en relación con la descripción y argumentación de una situación general o específica de un texto leído.

\section{F. Anuncio de una historia}

Se comienza comentando por parte del docente con sus estudiantes varios detalles y características de una historia o libro que hayan leído recientemente todo el grupo de clase. Seguidamente, en subgrupos, se les sugiere que elaboren una presentación en alguna de los programas o herramientas tecnológicas que lo posibilitan, en los que se anuncien las características más importantes de la obra escogida. De tal manera que puedan promocionar su lectura a otros estudiantes y amigos, y empleen para su intercambio el chat, foros, correos u otras plataformas. No olvidar explicar los principios de un buen 
anuncio, de igual manera estimular a realizar anuncios de otras obras y a coleccionarlos. De tal manera, que al final del curso puedan realizar una exposición de los mismos.

Todo el proceso en si requiere de un seguimiento y evaluación que se ajusta a las diversas modalidades o tipos de evaluación que el docente puede aplicar.

Se señala que la evaluación debe ser considerada como un medio para establecer medidas de corrección de los errores cometidos, ya sea en el proceso de enseñanza (en el que también hay muchos errores) o en el aprendizaje del estudiante. Lamentablemente, la evaluación a menudo se convierte en un fin en sí misma, perdiendo esta manera su potencialidad de cara al progreso de los estudiantes en sí.

Se debe tener en cuenta que la comprensión lectora precisa de una serie de procedimientos internos que son aquellos que permiten que la comprensión de lo leído tenga lugar. Por lo que estos procedimientos en el desarrollo de la estrategia y otras acciones y actividades que se pueden realizar serán los principales aspectos que deberán ser sujetos de evaluación.

Los aspectos que se consideran claves en el momento de evaluar la comprensión lectora son los siguientes:

La integración coherente de la información. Esta resulta, la habilidad más importante, ya que es la que nos permite entender las relaciones que se establecen entre los distintos contenidos textuales y por tanto, llegar a una comprensión plena del texto.

El grado de adecuación de la modalidad lectora a la intencionalidad de ésta.

El seguimiento de instrucciones. Es decir, la capacidad de entender instrucciones más o menos complejas en función de su edad y curso.

Los procesos de autocontrol de la comprensión lectora. Estos son aquellos que permiten darse cuenta de que no se ha comprendido correctamente algún aspecto o parte del texto. Al mismo tiempo, que permite usar una serie de recursos para solucionar las problemáticas existentes. Los procesos de autocontrol principales son los siguientes: 
- Detección de errores en la propia comprensión

- Recursos para resolver estos errores

- Recursos para extraer significados desconocidos

- Recuerdo de la información a largo plazo. El recuerdo de esta información al cabo de unos días indicará el nivel de la comprensión de contenidos determinados, ya que supondrá una modificación de sus conocimientos sobre la temática.

Otros aspectos relevantes y que a menudo no son tenidos en cuenta son los siguientes:

Utilización eficaz de los conocimientos previos. Es decir, la capacidad para utilizar aquella información que ya se conoce sobre la temática en cuestión, los que facilitan la comprensión del texto, y paralelamente permiten modificar los conocimientos sobre la temática en cuestión.

Utilización eficaz de las señales y ayudas del propio texto como elementos gráficos y de discurso.

Finalmente, más que centrarse en la evaluación de la comprensión lectora, el principal propósito es que el docente o miembro familiar (padres fundamentalmente) ejercite con frecuencia cada uno de las propuestas y retroalimente la forma de utilizarlas; de esta manera, se estará influyendo significativamente en el desarrollo intelectual del estudiante y en consecuencia en su desempeño escolar.

En todo este desarrollo el empleo de las TIC contribuye notablemente en la mejora de las actividades académicas de los estudiantes del lugar donde se realizó el estudio; esto corrobora los resultados obtenidos; además se concluyó que el empleo de la estrategia garantizará mayor motivación en cuanto a la participación, lo que es evidente también, al emplear las herramientas tecnológicas más requeridas para estas actividades, por ejemplo, el software Prezi.

La misma Prezi On Line manifiesta la serie de novedades que se pueden implementar a nivel didáctico, lo que se caracteriza por la organización, secuencialidad, novedad y participación de quienes acceden a esta tecnología, más aún en la mejora de la comprensión lectora; es necesario que se desarrolle la meta-comprensión, lo que, por la naturaleza de los procesos se puede trabajar con el software propuesto. 
Prezi On Line sostiene cualquier propuesta que sea sistematizable, porque además es integral y horizontal, y en este sentido, favorece que el estudiante sea consciente de los procesos que ha seguido en la comprensión lectora para reutilizarlos con las variantes que convengan. Por ejemplo, se sugieren utilizar los que a continuación se proponen en la Tabla \# 1.

\begin{tabular}{|c|c|c|c|}
\hline & & $\begin{array}{l}\text { GE: Alta } \\
\text { Implicación } \\
\text { de TIC }\end{array}$ & $\begin{array}{l}\text { GC: Baja } \\
\text { Implicación } \\
\text { de TIC }\end{array}$ \\
\hline \multirow{7}{*}{ Blackboard } & Correo & $\mathrm{x}$ & $x$ \\
\hline & $\begin{array}{l}\text { Videos } \\
\text { embebidos }\end{array}$ & $\mathrm{x}$ & \\
\hline & Evaluaciones & $\mathrm{x}$ & $\mathrm{x}$ \\
\hline & Tareas & $\mathrm{x}$ & \\
\hline & Foro & $x$ & $\mathrm{x}$ \\
\hline & $\begin{array}{l}\text { Chat o } \\
\text { Messenger }\end{array}$ & $\mathrm{x}$ & \\
\hline & Enlaces Web & $x$ & $x$ \\
\hline \multirow{2}{*}{ Internet } & Blogs & $x$ & \\
\hline & Bases de datos & $x$ & $\mathrm{x}$ \\
\hline \multirow{2}{*}{ Software } & CMapTools & $x$ & \\
\hline & Publisher & $x$ & \\
\hline \multirow{4}{*}{ Dispositivos } & iPads & $x$ & \\
\hline & $\begin{array}{l}\text { Cámaras } \\
\text { digitales }\end{array}$ & $\mathrm{x}$ & \\
\hline & Portátiles & $x$ & \\
\hline & Videobeam & $\mathrm{x}$ & $\mathrm{x}$ \\
\hline
\end{tabular}

Tabla \# 1. Nivel de implicación de TIC en el desarrollo de la comprensión lectora. Fuente: De Castro, A. E. et. Al. (2014).

\section{Materiales y métodos}

El proceso investigativo que se efectuó es de tipo descriptivo, donde se asume un enfoque mixto, con un diseño por etapas, en la cual, como estrategia metodológica asumida en correspondencia con el problema planteado, se orientó el proceso investigativo desde la complementariedad de elementos cuantitativos y lo cualitativos. 
Se efectuó fundamentalmente teniendo en cuenta la perspectiva de campo, ya que a través de esta modalidad se realizó la aplicación de una serie de instrumentos derivados de métodos científicos en la naturaleza educativa de una institución de la Educación Básica General de Ecuador, donde se trabajó con los involucrados en donde se ha detectado el problema. Es decir, en la escuela "Medardo Alfaro De Montecristi” de Portoviejo, con los estudiantes de sexto año de nivel Educación General Básica, en lo cual se obtuvo así información real y verídica que ayudó en el estudio, para poder brindar una solución tentativa.

Como unidad de análisis de la presente investigación se consideró a los estudiantes de sexto año de la Educación Básica General de la Escuela “Medardo Alfaro de Montecristi”, Manabí, Ecuador, y a los docentes que trabajan co9n ese año de dicha institución. Se asumió como población los (5) docentes de sexto año de la Educación Básica General de la Escuela “Medardo Alfaro de Montecristi”, Manabí, Ecuador y a (25) estudiantes del sexto año de la mencionada institución escolar.

Como muestra se consideró la representativa tomada de cada uno de los grupos declarados en la población que atiende y forma parte directamente en el desarrollo de las asignaturas en función de la comprensión lectora. Esta responde a la totalidad de los docentes que trabajan con el sexto año de la Escuela "Medardo Alfaro de Montecristi", Manabí, Ecuador (5) y el 100 \% de la totalidad de los estudiantes de sexto año de dicha institución.

El tipo de muestreo a realizar es aleatorio simple.

Los métodos teóricos utilizados, son los siguientes:

El método histórico-lógico, el método analítico - sintético, el método inductivo - deductivo. Como métodos empíricos se utilizaron:

$>$ La observación científica, el análisis documental, encuestas y entrevistas y método Delphi o criterio de experto.

Como técnica de recolección de información, se utilizó la prueba pedagógica, para complementar los resultados logrados y determinar el nivel de satisfacción de los principales usuarios de la propuesta elaborada.

Entre los métodos o técnicas estadísticas utilizadas se encuentran el cálculo de coeficiente de concordancia de Kendall y la prueba de hipótesis no paramétrica de los rangos con signo de Wilcoxon. 


\section{Resultados y discusión}

En tal sentido, el nivel de efectividad de la estrategia didáctica propuesta para el desarrollo de la comprensión lectora con el empleo de TIC, se realiza un experimento en su modalidad preexperimento, en el cual se aplican un pretest y un postest con mediciones temporales y sistemática a partir de varios instrumentos aplicados.

Como principal objetivo de este método aplicado se plantea: comprobar en la práctica pedagógica la efectividad de la estrategia didáctica para el desarrollo de la comprensión lectora con el empleo de TIC y el nivel de aprendizaje en los estudiantes.

La muestra con que se desarrolló el pre-experimento fue de 25 estudiantes de sexto año de la Educación General Básica, Montecristi, durante el curso escolar 2018 - 2019.

La observancia de las etapas de antes, durante y después en el acto de la lectura, como esencia en el desarrollo de la estrategia didáctica diseñada, con actividades y acciones que se emplean a partir del uso de TIC, significa que mediante la ejecución de esta se apropien de los conocimientos necesarios en función de la comprensión lectora.

El pre-experimento es aplicado en el grupo A del 6to año de la escuela "Medardo Alfaro de Montecristi” del Cantón Montecristi correspondiente a la Educación General Básica, el cual tiene una matrícula de 25 estudiantes y la influencia directa de 5 docentes. La totalidad de los estudiantes se encuentran en plena correspondencia con la edad cronológica y el año que cursan. Los docentes poseen una experiencia promedio de 7 años en el sector educacional.

Los momentos o etapas que determinan la estructura del pre-experimento pedagógico se desarrollan a través de procedimientos, los que constituyen los medios experimentales:

Experimento de constatación

El pre-experimento se inicia con un experimento de constatación o constatación del estado inicial, en que se encuentra la variable dependiente, en la cual en la etapa diagnóstica de la investigación fue realizado. Con esta se pretende verificar el nivel existente en torno al aprendizaje de la comprensión 
lectora en los estudiantes. También en cuanto a la calidad de sus interpretaciones y argumentaciones sobre los textos leídos.

Los resultados de la prueba pedagógica inicial que se aplica demuestran resultados deprimidos en cuanto a:

$>$ Significativa representatividad de los estudiantes en no realizar una primera lectura en voz baja para garantizar la familiarización con el texto.

$>$ Vocabulario pobre ya que algunas palabras tratadas y que deben conocer fueron identificadas como de difícil comprensión por los estudiantes

$>$ El conocimiento sobre el argumento del texto leído fue muy bajo, por lo que muy pocos afirmativamente respondieron sobre determinados aspectos como antecedentes del acontecimiento relatado.

$>$ Se revelaron problemas con la interpretación de la situación central del texto leído, es decir, los significados y las causas de la realización del acontecimiento tratado.

$>$ Muy pocos demostraron interés por profundizar en el acontecimiento tratado en el texto, recurriendo a la internet en busca de más información.

En relación con la hipótesis formulada y con el objetivo del experimento, se realizan las siguientes tareas experimentales:

a) Establecer las condiciones y exigencias didácticas necesarias para estructurar el eslabón de la dinámica del proceso de enseñanza -aprendizaje con respecto al empleo de TIC en función del desarrollo de la comprensión lectora en los estudiantes, cuyo cumplimiento contribuirá a perfeccionar la sistematización de este aparte y responde a la estrategia desplegada.

b) Comprobar el nivel de transformación del aprendizaje de la comprensión lectora y la actitud del estudiante ante el desarrollo de actividades con el empleo de TIC, en el cumplimiento de las exigencias de un aprendizaje consciente y activo.

Experimento de control:

Se concluye la etapa experimental con la constatación del estado final (experimento de control) en que se encuentra la variable dependiente, para verificar el nivel de aprendizaje logrado con la aplicación de la estrategia didáctica propuesta. 
Se aplica en tal sentido la posprueba, la cual evaluará los mismos elementos tratados en la preprueba pero con otro texto y adicionándole incluso otras preguntas de mayor nivel.

En la prueba de hipótesis para comparar los resultados del pre-experimento con la utilización del sistema estadístico SPSS V15.0, se definen dos variables con los datos correspondientes al aprendizaje de la comprensión lectora desde el empleo de TIC. La variable relacionada con la medida se expresa en una escala de intervalos, y se realiza un análisis antes y después de aplicar el pre-experimento a un total de 25 estudiantes, por lo que se utiliza la prueba estadística no paramétrica de los rangos con signos de Wilcoxon.

La probabilidad asociada al valor de $\mathrm{Z}=-4,038$, con una probabilidad $\mathrm{p}=0,0$. Como $\mathrm{p}<\alpha$, entonces se rechaza la hipótesis $\mathrm{H} 0$; es decir, se acepta H1, por lo que se puede plantear, con un nivel de confiabilidad del $99 \%$, que los resultados obtenidos en el pre-test y en el post-test difieren significativamente. Dado que la media de la evaluación del aprendizaje de la comprensión lectora en los estudiantes de 6to año es 3,0 muy superior al obtenido al inicio $(1,50)$, entonces queda demostrado, al $99 \%$ de confianza, que los resultados obtenidos en el post-test son mejores que los obtenidos en el pre-test y estos difieren significativamente desde el punto de vista estadístico, por lo que los resultados que se alcanzan no se deben al azar, sino a la efectividad de la aplicación de la estrategia.

Existen diferencias significativas entre la frecuencia de errores en el diagnóstico inicial y el final, a favor del diagnóstico final.

Se demuestra además que la ejecución de actividades y acciones realizadas en el propio desarrollo de la estrategia didáctica en el periodo establecido, propició considerablemente el interés y la motivación por la lectura, esto en mayor medida acentuado y posibilitado por el empleo de TIC, de herramientas y programas que de conjunto se utilizaron en la ejecución de la misma.

Al efectuar la adecuada interpretación de las ideas centrales y los argumentos de los textos tratados, así como todos los pasos y procedimientos a seguir para lograr una adecuada comprensión lectora, se suceden y obtiene resultados de aprendizajes positivos. 
La observancia en el propio desarrollo de la lectura y las respuestas ofrecidas por los estudiantes en torno a lo leído, demostró mejoras en el desarrollo de la comprensión lectora en ellos.

Durante la aplicación del pre-experimento se demuestra que:

$>$ La información expresa de cómo se ha venido desarrollando la comprensión lectora en una escuela de la Educación Básica General en Portoviejo, Ecuador, es efectiva en tanto permite potenciar la elaboración e innovación en acciones y actividad para seguir consolidando la comprensión lectora en los estudiantes, así como el empleo de diversas herramientas y recursos tecnológicos para fomentar y complementar el trabajo en esta dirección.

$>$ La correcta selección de textos que favorezcan un nivel ascendente de análisis, interpretación y expresión de ideas en los argumentos que ofrece el texto, el equilibrio de los procedimientos y el empleo de materiales didácticos de conjunto con herramientas y programas tecnológicos que contribuyan a potenciar las actividades y acciones programadas en la estrategia didáctica. Además, garantiza la sistematicidad, consecutividad, solidez, motivación y voluntad para el desarrollo del aprendizaje en este sentido.

> La aplicación de la estrategia didáctica con el empleo de TIC para el desarrollo de la comprensión lectora, favorece en general el aumento de la necesidad y la motivación en el estudiante por la lectura, hasta alcanzar en el último período del curso mejores niveles en la expresión e interpretación de las ideas explicitas e implícitas en los textos leídos.

De forma general la aplicación de la estrategia didáctica propicia que el estudiante durante un sistema de clases, pueda prepararse con mayores posibilidades de profundizar en los textos y establecer interacciones sistemáticas con docentes y otros miembros de su grupo, al utilizar las TIC con ese objetivo, lo que de forma significativa incrementa la cantidad de textos y ejercicios de lectura.

Proporciona también una correcta evaluación del cumplimiento del objetivo propuesto, por eso es importante establecer los criterios o exigencias que deben cumplimentar los estudiantes en cada tarea de aprendizaje y en ello enfatizar para que los tengan siempre presentes en la medición del nivel alcanzado.

Permite la solidez y perdurabilidad del conocimiento, lo que sienta las bases para no ocasionar interrupciones en la producción de significados en los textos leídos. El orden lógico en la exposición 
de las ideas sin que se afecten los significados y mucho menos la creatividad al desarrollar otras ideas correspondientes y relacionales.

La validez de la aplicación de la estrategia didáctica también pudo valorarse a partir de otros aspectos importantes:

1. Calidad de la clase donde se efectuaron actividades y acciones relacionadas con la comprensión lectora, denotan el nivel de implicación del estudiante en la búsqueda del conocimiento a partir del diseño y organización de las tareas montadas en herramientas y programas propios de las TIC, para una mejor y mayor atención y aprovechamiento de la unidad entre el aspecto cognitivo y el motivacional, así como la concreción de acciones y operaciones que lleven a la formación de las habilidades para el logro adecuado de la comprensión lectora.

2. Preparación teórico-metodológica de los docentes de la educación general básica, para el tratamiento a la comprensión lector de los estudiantes de 6to año en el utilización de las TIC en el desarrollo de la comprensión lectora, en la producción de significados e interpretación de códigos e ideas de los textos leídos.

3. Nivel alcanzado en el desarrollo y utilización de las habilidades para la comprensión lectora en el desarrollo del aprendizaje por parte de los estudiantes, y por parte del docente en nuevas formas, acciones y procedimientos desde una estrategia didáctica y el empleo de TIC para ello.

La adopción de esta estrategia didáctica y acciones que se proponen con el empleo de TIC para el desarrollo de la comprensión lectora, contribuye a elevar la motivación de los estudiantes en la ejecución de las actividades y a interpretar las ideas de los textos leídos. También de nuevos recursos para el desarrollo de este tipo de actividades por parte de los docentes y su manejo por parte de los estudiantes.

\section{Conclusiones}

Los principales elementos teórico-conceptuales del proceso de enseñanza-aprendizaje relacionado con la comprensión lectora en el 6to año de la Educación General Básica, y todo lo concerniente con el empleo de TIC como vía de desarrollar las actividades y los contenidos, facilita el conocimiento de 
las causas y relaciones causales entre las insuficiencias que se presentan en torno a ellos de acuerdo con las exigencias existentes para los objetivos del año y demás metas a cumplimentar.

Como parte de la estrategia didáctica, constituyen elementos novedosos la revelación de un conjunto de acciones que se integran a las etapas y procedimientos sobre el proceso de desarrollar la compresión lectora, sistema de relaciones que se dan entre sus subsistemas en este proceso, incorporando además el empleo de varias TIC, herramientas y programas como el Prezi, que favorecieron la motivación y el interés de los estudiantes por lo realizado, como regularidad esencial.

Se constata la pertinencia y validez de los aportes propuestos como resultados de la investigación, lo cual se evidencia en resultados positivos en cuanto al aprendizaje de los estudiantes sobre la comprensión lectora, sistematización de las habilidades lectoras y mejoras en el vocabulario y motivación por la lectura.

\section{Referencias bibliográficas}

1. Álvarez, G. (2012). Entornos virtuales de aprendizaje y didáctica de la lengua: dos experiencias con integración de TIC para mejorar las habilidades de lectura y escritura de estudiantes preuniversitarios. Revista Q: Educación Comunicación Tecnología, 6(12), 1-23

2. Ariaz, G. J., Severiche, W. O., Gómez, E. P., Boneth, E. R., \& Arévalo, L. C. C. (2018). Pertinencia de las tecnologías de la información y la comunicación para el fortalecimiento de la comprensión lectora. IJMSOR: International Journal of Management Science \& Operation Research, 3(1), 57-63.

3. Campos Lizonde, O. (2020). TIC'S y comprensión lectora en estudiantes de quinto grado de primaria de la Institución Educativa 6062 de Villa El Salvador, 2019.

4. Celin Galban, A. L., \& Mendoza Lubo, S. C. (2019). Textos y audios digitales, dos medios tecnológicos eficientes para potencializar la competencia lectora (Doctoral dissertation, Universidad de la Costa).

5. De Castro, A. E., Oliveros, M. O. C., Carbonó, V. I., Robles, H. S., Plaza, D. D., Florez, D. G., ... \& Álvarez, S. (2014). Comprensión lectora y TIC en la universidad. Apertura, 6(1), 46-59.

6. Echevarría, M. (2002). Programas computacionales para el español como lengua materna. Revista signos: estudios de lingüística, (51-52), 163-194. 
7. Tamayo Mendoza, R. M. (2018). El uso de las Tics y su influencia en el desarrollo de la comprensión lectora en los estudiantes del 6to grado de educación primaria de la IE Nestor Escudero Otero UGEL 05-2017, del distrito de San Juan de Lurigancho.

8. Thorne, C., Morla, K., Uccelli, P., Nakano, T., Mauchi, B., Landeo, L., ... \& Huerta, R. (2013). Efecto de una plataforma virtual en comprensión de lectura y vocabulario: Una alternativa para mejorar las capacidades lectoras en primaria. Revista de Psicología (PUCP), 31(1), 3-35.

9. Tresca, M. (2015). Comprensión de textos. Obtenido de http://www.uca.edu.ar/uca/common/grupo18/files/Comprension_de_textos.pdf 